\title{
Trauma and BMI Mortality
}

\author{
Tejal S. Brahmbhatt ${ }^{1} \cdot$ Michael Hernon $^{1} \cdot$ Charles Jeffrey Siegert $^{1} \cdot$ Leneé Plauché $^{1}$ • \\ Lorrie S. Young ${ }^{2} \cdot$ Peter Burke $^{1}$
}

Published online: 25 May 2017

(C) Springer Science+Business Media New York 2017

\begin{abstract}
Purpose of Review Obesity has been recognized as an impactful comorbid condition. It has been demonstrated to be an independent risk factor for morbidity and mortality following trauma.

Recent Findings Multiple studies and reviews have strongly suggested obesity to be associated with increased risk for postinjury morbidity and mortality. As obesity is associated with a pro-inflammatory state, it has been suggested to negatively affect the respiratory, cardiovascular, coagulation, and renal systems and the ability and manner in which an obese patient heals.

Summary As obesity continues to rise at alarming rates in the USA, this population of patients requires special consideration
\end{abstract}

This article is part of the Topical Collection on Obesity Treatment

Tejal S. Brahmbhatt

Tejal.Brahmbhatt@bmc.org

Michael Hernon

Michael.Hernon@bmc.org

Charles Jeffrey Siegert

Charles.Siegert@bmc.org

Leneé Plauché

LPlauche@bu.edu

Lorrie S. Young

Lorrie.Young@bmc.org

Peter Burke

Peter.Burke@bmc.org

1 Boston University School of Medicine, 840 Harrison Ave, Dowling 2 South, Boston, MA 02118, USA

2 Boston Medical Center, Instructor of Medicine, 717 Albany St, Boston, MA 02118, USA and continued research is underway to delineate relationships between obesity, trauma, and outcomes in an effort to improve overall care.

Keywords Obesity $\cdot$ Comorbid condition $\cdot$ Trauma $\cdot$ BMI · Morbidity $\cdot$ Mortality

\section{Introduction}

The number of individuals suffering from obesity, as defined by a body mass index (BMI) of 30 or higher, has been increasing at an alarming rate over the last 50 years in the USA. Complications in the management and recovery from blunt and penetrating trauma have been shown to have an independent association with obesity. As obesity leads to a number of physiological and anatomical changes, the body's response to injury is directly affected in injury. It has been reported that obese patients have an increase of respiratory, cardiovascular, venous thromboembolic, and infectious complications.

\section{Penetrating Trauma}

\section{Epidemiology}

Penetrating trauma (PT) accounts for approximately $15 \%$ of total injury-related disease on a global spectrum, not accounting for those involved in military engagement, though rates can reach as high as $20-45 \%$ in some urban areas [1]. These high-impact regions account for up to $80 \%$ of all PT and also tend to be associated with low socioeconomic status and high rates of substance abuse [1].

Victims of PT are more likely to be younger than those of blunt trauma (BT), on average 31.4 versus 46.1 years old in 
the BT population [2]. They are also more likely to be male, representing up to $80-86 \%$ of PT patients [1,2], though rates of PT in women have been shown to increase with BMI [3••]. Gunshot wounds (GSW) are the most common vehicle of PT and also the most lethal, representing up to $45 \%$ of all trauma deaths in some trauma centers [2]. According to a 2012 National Institute of Health study, even in children \& adolescents the prevalence has increased over the last decade [4, 5], with the prevalence of obesity at major trauma centers reflecting that of the general population [6]. Current literature is conflicting regarding the distribution of injury mechanism in obese and non-obese cohorts-some suggest no difference in injury mechanism [6] while others have demonstrated the obese population is more likely to present with PT (34.8\% obese versus $22.5 \%$ non-obese) [7].

While the rates of computed tomography imaging performed on obese PT victims versus their non-obese counterparts are similar at a rate of $35 \%$, the rates of non-therapeutic surgical intervention, defined as an operation that does not identify or require repair of injured structures, increased significantly with BMI $[3,8]$. In the general population, rates of non-therapeutic operations range between 23 to $53 \%$ for stab wounds (SWs) and 5.3 to $27 \%$ for GSWs [8-10], while studies evaluating rates of non-therapeutic operation across BMI groups showed rates increase with BMI and reach as high as 75\% (BMI 29-30, 42\%; BMI 30-35, 52\%; BMI >35, 75\%) [3].

\section{Pathophysiology}

The impact of obesity on the management and overall outcomes in abdominal trauma poses a significant challenge for the healthcare workforce, particularly in the setting of increasing rates of obesity at epidemic proportions. Multiple explanations exist for the role of obesity in the development of various post-operative complications and the pathogenesis of wound healing.

Obese patients typically carry a medical history of multiple comorbidities, potentially including diabetes, hypertension, cardiovascular disease, arrhythmia, liver dysfunction, asthma, and chronic obstructive pulmonary disease, all of which predispose this population to poor outcomes even for an elective operation [11]. Obesity brings challenges to perioperative management and intensive care that include difficult airway, increased requirement of bedside care for proper hygiene and mobilization, and technical difficulties of placing peripheral intravenous lines that leads to higher rates of central venous lines and subsequent risks of bacteremia $[12,13]$.

In the trauma patient, obesity has been found to be an independent risk factor for post-injury organ dysfunction that can be explained by a systemic pro-inflammatory state resulting in increased circulating levels of inflammatory mediators [14-16]. Obese patients have also been shown to have increased susceptibility to ischemia-reperfusion injury secondary to thinner bowel wall and intestinal vessels [15]. They are more likely to require delayed closure of the abdominal wall after laparotomy secondary to increased vascular permeability resulting in substantially worse bowel wall edema than that seen in non-obese populations [15]. Insulin resistance in obesity is also known to impact wound healing and surgical site infection rates [14]. Additionally, poor baseline nutritional status and high rates of metabolic acidosis play a role in the challenging physiology of wound healing in the obese population $[14,17,18]$.

In addition to these pathophysiological processes predisposing obese patients to worse overall outcomes, it has been shown that obese trauma patients suffer distinct injuries from that of their non-obese counterparts. Interestingly, obesity has been shown to have a protective effect in penetrating trauma secondary to an increased depth of pre-peritoneal fat, which results in lower rates of peritoneal violation, particularly in victims of SWs [3, 19]. Whether the role of obesity in GSWs is protective is not as clear and requires further investigation.

The obese patient also presents unique diagnostic and anatomical challenges. The Focused Assessment with Sonography in Trauma exam, a widely used diagnostic method for determining the need for surgical intervention in trauma using ultrasound, faces significant limitations in the obese population due to fat attenuation of ultrasound waves and a resultant poor image quality secondary to failure of the wave to penetrate the pre-peritoneal fat layer [17, 20]. One study describing this limitation explains that a $7-\mathrm{MHz}$ ultrasound transducer will attenuate $50 \%$ traveling through $1 \mathrm{~cm}$ of fat, resulting in $94 \%$ total attenuation in a pre-peritoneal fat layer of $8 \mathrm{~cm}$ depth [20]. These authors suggest selecting the lowest frequency transducer for best image quality in the obese patient and support the technique of tissue harmonic imaging which induces harmonic sound waves and employs the properties of fat to improve image quality [20]. From a surgical standpoint, a large pannus distorts anatomical landmarks, displacing the distance of the umbilicus from the true vertical midpoint by an average of $3.5 \mathrm{~cm}$ (compared to $0.87 \mathrm{~cm}$ in patients of normal BMI) which can impact surgical strategies such as incision site and laparoscopic port placement [17].

\section{Morbidity}

It is well established within the medical literature that obese trauma patients have worse outcomes despite no statistically significant difference in injury severity score (ISS) [12, 14], an anatomical injury scoring system which correlates linearly with morbidity, mortality, and hospital length of stay (LOS) [21]. A subcategory of the ISS is the abbreviated injury score (AIS), which looks at individual anatomical regions. 
Few studies evaluate the impact of obesity exclusively in penetrating trauma. However, one study evaluating stab wounds in obese patients found that abdominal AIS scores decrease with increasing BMI, demonstrating that obesity in fact plays a protective role, despite no difference in overall ISS [3]. An association also exists between increased BMI and decreased rates of peritoneal violation, visceral injury, and injury requiring therapeutic surgical intervention [3]. This study showed rates of operative management 3.4 times higher in the thinnest group when compared to the most obese group (67 versus $20 \%)$ [3].

While this protective theory has been supported in the literature in terms of severity of injury in abdominal trauma, when controlling for injury severity, obese patients irrespective of mechanism of injury experience worse overall outcomes including increased ventilator days, central line days, and urinary catheter days, which lend respectively to a twofold increased rate of respiratory infections, bacteremia, and urinary tract infections $[3,12]$. Obesity has also been shown to be associated with increased post-operative complications, LOS, intensive care unit days (ICU), ventilator days, and overall mortality $[3,7,14]$.

Though few studies on the obese trauma population stratify their data by mechanism of injury, those that do suggest that in PT, ICU days in fact decrease as a function of increasing BMI and that there is no association between BMI and LOS $[3,7$, 22]. One study evaluating stab wounds in the obese population found that the rates of severe injury, defined as an ISS $>25$, decreased as a function of BMI, as did the rates of visceral injury. This same study identified small bowel as the most commonly injured viscus in obese patients, likely as a factor of the anterior positioning of the organ and depth of the wound being limited to the length of the knife blade [3].

\section{Mortality}

The tri-modal distribution of mortality in trauma is a welldescribed model detailing the temporal relationship of mortality in major trauma. This model attests that mortality can be grouped into three peaks: $<60 \mathrm{~min}, 1-4 \mathrm{~h}$, and $>7$ days postinjury, associated with mortality rates of 45,34 , and $20 \%$, respectively [2]. Recent literature suggests however that this model is not applicable to a mature trauma system with seasoned pre-hospital services and trauma centers, which have instead demonstrated a bimodal distribution of mortality from penetrating trauma of $63.7 \%$ within $60 \mathrm{~min}$ of injury (with $24.8 \%$ occurring at the scene) and $2.6 \%$ between 1 and $6 \mathrm{~h}$ post-injury, with complete elimination of the third peak [2]. The tri-modal distribution of mortality has not been studied specifically in the obese population and so it remains unclear whether elimination of the third peak would apply to this population.
A meta-analysis evaluating the impact of obesity on mortality in all trauma found an odds ratio (OR) of 1.45 in the obese versus the non-obese trauma patient despite no difference in ISS, with acute respiratory distress syndrome (ARDS), acute renal failure (ARF), and multiple organ failure (MOF) being the most common and most fatal complications in the obese patient [14].

However, there exist conflicting pools of thought regarding the impact of obesity on morbidity in PT specifically. In the general population, penetrating trauma accounts for approximately 15 to $20 \%$ of admissions but is responsible for half of the fatalities [2]. When stratified by mechanism of injury, fatalities from penetrating trauma are more commonly associated with significant injury to the chest and abdomen, both anatomical regions which have increased depth of subcutaneous fat in the obese patient [2]. One study found that, when stratified by weight, victims of penetrating trauma with body weight at or above the 96th percentile had a two to fourfold increased mortality rate than the general population [6]. Despite this, some studies report no significant difference in mortality between the obese and non-obese (10.6 versus $10.2 \%, p=0.9$ ) [7]. If obesity is in fact protective, it is surprising that this protective effect is not reflected by a decrease in mortality.

One of these studies that did not find a difference in mortality suggested a theory that their findings might be related to the fact that their study was performed at a major bariatric surgery center, suggesting that experience with the unique physiology of the obese patient can improve outcomes, a position echoed by others who argue for the development of bariatric trauma centers and the need for a unique management algorithm for obese patients in the Advanced Trauma Life Support curriculum [7, 17].

\section{Blunt Trauma}

\section{Epidemiology}

The prevalence of obesity in the USA continues to rise over the past few decades. The Center for Disease Control has now published that in 2014, more than one-third of adults are considered obese [23]. Obesity rates continue to rise in the pediatric population also with approximately $17 \%$ of children aged 2 to 19 years old are classified as being obese. The rising prevalence of obesity in the USA poses an even greater challenge in the care of the obese trauma patient. Trauma continues to be the number one causal agent of mortality in the USA in the age group of 1 to 45 years old.

Providing care for the obese patient poses a significant burden and challenge even at highly specialized trauma centers [24•]. These challenges include the physical treatment of the patient and the economic burden of caring for this population. 
The economic burden resulting from trauma is profound, costing the USA 671 billion dollars annually in combined health care costs and loss of productivity [24-]. This is attributed to the need for more ancillary staffing, specialized equipment, higher rates of complications, higher level of care, longer LOS, and need for rehabilitation.

The type of traumatic injuries following blunt trauma in the obese population seems to have a more pattern distribution in comparison with the rest of population. Multiple studies have shown that blunt trauma patients with a BMI $>30$ have a substantially greater incidence of chest trauma resulting in higher rates of fractured ribs and pulmonary contusions resulting in significant pulmonary complications and mortality [25]. Other studies showed that patients with high body mass index were more likely to sustain pelvic fractures [26]. Both of these studies have also showed that higher BMI patients are less likely to sustain intracranial or blunt abdominal injuries.

\section{Pathophysiology}

The pathophysiology behind these types of injury patterns is still being debated. The presumed "cushion effect" has been debated and believed to be debunked in some forms of injury including bony injuries [19]. Traditional teaching has stated that an increased body mass leads to increased bone density portending more resilience with fractures; however, recent studies have reported more fat deposits in bone related to obesity resulting in weakening and more susceptibility in fracture. The belief that kinetic energy is the form of energy transfer which results in associated injuries. Kinetic energy is proportional to mass meaning that obesity increases energy transfer and possibly severity of injury.

\section{Morbidity and Mortality}

Morbid obesity is an independent risk factor for death following blunt trauma. There are numerous reasons why obesity may affect outcomes of blunt trauma. Obesity not only alters the pattern of injury, but it also increases the incidence of certain in-hospital complications. Just as obesity plays a role in other diseases such as heart disease and diabetes, so does obesity create new hurdles for the care of the blunt trauma patient.

The initial injury profile of obese patients following blunt trauma reveals an increased rate of chest and extremity injuries yet fewer head injuries [27]. And yet, despite the lower rates of head injury in obese patients, there are still an increased number of ventilator days and worse outcomes.

Much of the data regarding the complications of obesity in blunt trauma is derived from the orthopedic experience with extremity fractures. In looking at tibia shaft fractures, there is an increase in pulmonary embolism, respiratory failure, acute renal failure, cardiovascular accident, deep vein thrombosis, orthopedic non-union, and infection [28]. Similarly, tibial shaft fractures were associate with increased morbidity and femoral shaft fractures were associated with higher mortality and ARDS [29].

Data from the National Trauma Data Bank, including over 32,000 patients, reveals that ARDS and pneumonia are the most common complications in obese patients [30]. It is debated as to whether this is a function of a higher incidence of chest trauma on initial presentation or whether the increased pulmonary tree resistance and decreased chest wall compliance contributes to worse respiratory physiology. The work of breathing is greater in the morbid obese, and thus any lung insult may be further potentiated.

The question remains as to whether the blunt injury pattern in obese patients determines the outcomes versus the higher rate of post-operative complications. The interplay of infection and/or sepsis with obesity is important to consider. Obesity is related to an increase in nosocomial infections, urinary tract, and surgical site infections. Antibiotic treatment failure or under dosing contributes to increased rates of infection in obese trauma patients. In this respect, proper dosing of antibiotics based on weight is a quick means to improve increased morbidity and mortality in obese trauma patients.

\section{Trauma-Associated Critical Illness}

Patients that are obese and suffering concomitantly from critical illness have been studied. Early single-center analyses suggest that both obese and non-obese trauma victims suffer the same surgical ICU length of stay [31]. This has been found similarly in meta-analyses of burn trauma victims, but this may in fact be due to the small number of patients being compared in the studies reported [32]. However, we do know that obese patients are at increased risk of post-injury multisystem organ failure [33, 34]. Of recent note, it has been reported that there is a negative impact on outcomes in critically ill obese patients leading to an increased ICU length of stay $[12,34,35]$. Suffering from obesity, there is more than a twofold increase in the relative risk of being admitted to the ICU after trauma as an independent predictor when controlled for age, ISS, and premorbid risk factors [12]. Recent conflicting data has been reported to suggest that obesity was not associated with an increase in mortality in the ICU [36]. Even though we do know that obesity is a chronic state of inflammation, one argument for why the differences may exist in outcome analyses is that the volume of adipose tissue may act as an adipokine factory that produces anti-inflammatory cytokines and hormones that could impact the immune response positively [37]. 


\section{Conclusion}

The number of obese patients affected by trauma in the USA is rapidly growing. It is becoming clear from the literature that blunt and penetrating trauma in the morbidly obese is associated with increased mortality, multisystem organ failure, acute renal failure, and increased ICU days. Just as we adjust our optics for children in the trauma bay, so to must we adjust our approach to the obese trauma patient. Further research should be pursued to delineate beyond the link between obesity and trauma to determine a more complete understanding of the pathophysiology so as to begin efforts to improve outcomes.

\section{Compliance with Ethical Standards}

Conflict of Interest Tejal S. Brahmbhatt, Peter Burke, Michael Hernon, Charles Jeffrey Siegert, Lorrie S. Young, and Leneé Plauché declare they have no conflict of interest.

Human and Animal Rights and Informed Consent This article does not contain any studies with human or animal subjects performed by any of the authors.

\section{References}

Papers of particular interest, published recently, have been highlighted as: • Of importance $\bullet \bullet$ Of major importance

1. Søreide K. Epidemiology of major trauma. Br J Surg. 2009;96(7): 697-8. doi:10.1002/bjs.6643.

2. Demetriades D, Kimbrell B, Salim A, Velmahos G, Rhee P, Preston $\mathrm{C}$, et al. Trauma deaths in a mature urban trauma system: is "trumodal" distribution a valid concept? J Am Coll Surg. 2005;201(3):343-8.

3.• Bloom MB, Ley EJ, Liou DZ, Tran T, Chung R, Melo N, et al. Impact of body mass index on injury in abdominal stab wounds: implications for management. J Surg Res. 2015;197(1):162-6. doi: $10.1016 /$ j.jss.2015.03.052. This is the most recent article summarizing most recent available radiographic modalities and non-operative management penetrating trauma.

4. Ogden CL, Carroll MD, Kit BK, Flegal KM. Prevalence of childhood and adult obesity in the United States, 2011-2012. JAMA. 2014;311(8):806-14. doi:10.1001/jama.2012.40.

5. Rana AR, Michalsky MP, Teich S, Groner JI, Caniano DA, Schuster DP. Childhood obesity: a risk factor for injuries observed at a level-1 trauma center. J Pediatr Surg. 2009;44(8):1601-5. doi: 10.1016/j.jpedsurg.2008.11.060.

6. Glance LG, Li Y, Osler TM, Mukamel DB, Dick AW. Impact of obesity on mortality and complications in trauma patients. Ann Surg. 2014;259(3):576-81. doi:10.1097/SLA.0000000000000330.

7. Alban RF, Lyass S, Margulies DR, Shabot MM. Obesity does not affect mortality after trauma. Am Surg. 2006;72(10):966-9.

8. Ball CG. Current management of penetrating torso trauma: nontherapeutic is not good enough anymore. Can J Surg. 2014;57(2):E36-43.

9. Velmahos GC, Demetriades D, Toutouzas KG, Sarkisyan G, Chan LS, Ishak R, et al. Selective nonoperative management in 1,856 patients with abdominal gunshot wounds: should routine laparotomy still be the standard of care? Ann Surg. 2001;234(3):395-402. discussion 402-3
10. Como JJ, Bokhari F, Chiu WC, Duane TM, Holevar MR, Tandoh MA, et al. Practice management guidelines for selective nonoperative management of penetrating abdominal trauma. J Trauma. 2010;68(3):721-33. doi:10.1097/TA.0b013e3181cf7d07.

11. Pikarsky AJ, Saida Y, Yamaguchi T, Martinez S, Chen W, Weiss $\mathrm{EG}$, et al. Is obesity a high-risk factor for laparoscopic colorectal surgery? Surg Endosc. 2002;16(5):855-8.

12. Bochicchio GV, Joshi M, Bochicchio K, Nehman S, Tracy JK, Scalea TM. Impact of obesity in the critically ill trauma patient: a prospective study. J Am Coll Surg. 2006;203(4):533-8.

13. Duchesne JC, Schmieg RE Jr, Simmons JD, Islam T, McGinness CL, McSwain NE Jr. Impact of obesity in damage control laparotomy patients. J Trauma 2009;67(1):108-112; discussion 112-4. Doi: 10.1097/TA.0b013e3181a92ce0.

14. Liu T, Chen JJ, Bai X, Zheng GS, Gai W. The effect of obesity on outcomes in trauma patients: a meta-analysis. Injury. 2013;44(9): 1145-52. doi:10.1016/j.injury.2012.10.038.

15. Haricharan RN, Dooley AC, Weinberg JA, McGwin Jr G, MacLennan PA, Griffin RL, et al. Body mass index affects time to definitive closure after damage control surgery. J Trauma. 2009;66(6):1683-7. doi:10.1097/TA.0b013e3181a4e818.

16. Lyon CJ, Law RE, Hsueh WA. Minireview: adiposity, inflammation, and atherogenesis. Endocrinology. 2003;144(6):2195-200.

17. Twaij A, Sodergren MH, Pucher PH, Batrick N, Purkayastha S. A growing problem: implications of obesity on the provision of trauma care. Obes Surg. 2013;23(12):2113-20. doi:10.1007/s11695013-1093-x.

18. Nelson J, Billeter AT, Seifert B, Neuhaus V, Trentz O, Hofer CK, et al. Obese trauma patients are at increased risk of early hypovolemic shock: a retrospective cohort analysis of 1,084 severely injured patients. Crit Care. 2012;16(3):R77. doi:10.1186/cc11334.

19. Arbabi S, Wahl WL, Hemmila MR, Kohoyda-Inglis C, Taheri PA, Wang SC. The cushion effect. J Trauma. 2003;54(6):1090-3.

20. Modica MJ, Kanal KM, Gunn ML. The obese emergency patient: imaging challenges and solutions. Radiographics. 2011;31(3):81123. doi:10.1148/rg.313105138.

21. Baker SP, O'Neill B, Haddon Jr W, Long WB. The injury severity score: a method for describing patients with multiple injuries and evaluating emergency care. J Trauma. 1974;14(3):187-96.

22. Osborne Z, Rowitz B, Moore H, Oliphant U, Butler J, Olson M, et al. Obesity in trauma: outcomes and disposition trends. Am J Surg. 2014;207(3):387-92. doi:10.1016/j.amjsurg.2013.10.013.

23. Johnson CL, Dohrmann SM, Burt VL, Mohadjer LK. National health and nutrition examination survey: sample design, 20112014. Vital Health Stat 2. 2014 Mar;(162):1-33.

24. Prevention Centers for Disease Control and Prevention, National Center for Injury Prevention and Control [Internet]. Web based Injury Statistics Query and Reporting System (WISQARS). 2016. This is a leading authority of national data regarding reported statistics for injury statistics.

25. Choban PS, Weireter Jr LJ, Maynes C. Obesity and increased mortality in blunt trauma. J Trauma. 1991;31(9):1253-7.

26. Boulanger BR, Milzman D, Mitchell K, Rodriguez A. Body habitus as a predictor of injury pattern after blunt trauma. J Trauma. 1992;33(2):228-32.

27. Brown CV, Neville AL, Rhee P, Slim A, Velmahos GC, Demetriades D. The impact of obesity on the outcomes of 1,153 critically injured blunt trauma patients. J Trauma. 2005;59(5): 1048-51.

28. Burrus MT, Werner BC, Yarboro SR. Obesity is associated with increased postoperative complications after operative management of tibial shaft fractures. Injury. 2016;47(2):465-70. doi:10.1016/j. injury.2015.10.026.

29. Weinlein JC, Deaderick S, Murphy RF. Morbid obesity increases the risk for systemic complications in patients with femoral shaft 
fractures. J Orthop Trauma. 2015;29(3):e91-5. doi:10.1097/BOT. 0000000000000167.

30. Ditillo M, Pandit V, Rhee P, Aziz H, Hadeed S, Bhattacharya B, et al. Morbid obesity predisposes trauma patients to worse outcomes: a National Trauma Data Bank analysis. J Trauma Acute Care Surg. 2014;76(1):176-9. doi:10.1097/TA.0b013e3182ab0d7c.

31. Neville AL, Brown CV, Weng J, Demetriades D, Velmahos GC. Obesity is an independent risk factor of mortality n severely injured blunt trauma patients. Arch Surg. 2004 Sept;139(9):983-7.

32. Sayampanathan AA. Systematic review and meta-analysis of complications and outcomes of obese patients with burns. Burns. 2016;42(8):1634-43. doi:10.1016/j.burns.2016.05.008.

33. Ciesla DJ, Moore EE, Johnson JL, Burch JM, Cothren CC, Sauaia A. Obesity increases risk of organ failure after severe trauma. J Am Coll Surg. 2006;203(4):539-45.
34. Akinnusi ME, Pineda LA, El Solh AA. Effect of obesity on intensive care morbidity and mortality: a meta-analysis. Crit Care Med. 2008;26(1):151-8.

35. Sakr Y, Madl C, Filipescu D, Moreno R, Groeneveld J, Artigas A, et al. Obesity is associated with increased morbidity but not mortality in critically ill patients. Intensive Care Med. 2008;34(11): 1999-2009. doi:10.1007/s00134-008-1243-0.

36. Wardell S, Wall A, Bryce R, Gjevre JA, Laframboise K, Reid JK. The association between obesity and outcomes in critically ill patients. Can Respir J. 2015;22(1):23-30.

37. Selim BJ, Ramar K, Surani S. Obesity in the intensive care unit: risks and complications. Hosp Pract (1995). 2016 Aug. 44(3):14656. Doi: 10.1080/21548331.2016.1179558. 